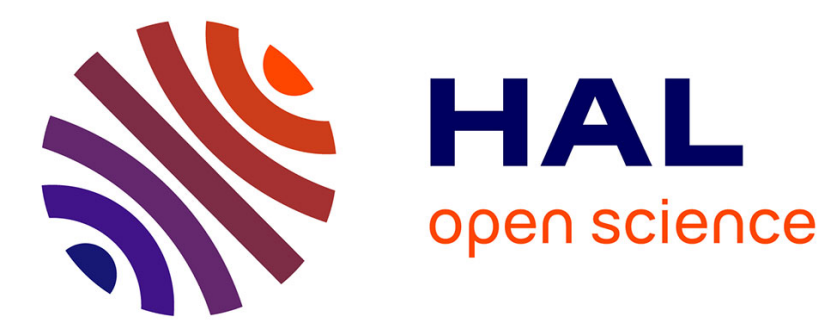

\title{
The Rotational Spectrum of the $\beta$-Cyanovinyl Radical: A Possible Astrophysical N-Heterocycle Precursor
}

\author{
Sommer Johansen, M. A. Martin-Drumel, Kyle Crabtree
}

\section{To cite this version:}

Sommer Johansen, M. A. Martin-Drumel, Kyle Crabtree. The Rotational Spectrum of the $\beta$ Cyanovinyl Radical: A Possible Astrophysical N-Heterocycle Precursor. Journal of Physical Chemistry A, 2019, 123 (24), pp.5171-5177. 10.1021/acs.jpca.9b03798 . hal-02305395

\section{HAL Id: hal-02305395 \\ https://hal.science/hal-02305395}

Submitted on 4 Oct 2019

HAL is a multi-disciplinary open access archive for the deposit and dissemination of scientific research documents, whether they are published or not. The documents may come from teaching and research institutions in France or abroad, or from public or private research centers.
L'archive ouverte pluridisciplinaire HAL, est destinée au dépôt et à la diffusion de documents scientifiques de niveau recherche, publiés ou non, émanant des établissements d'enseignement et de recherche français ou étrangers, des laboratoires publics ou privés. 


\title{
The Rotational Spectrum of the $\beta$-Cyanovinyl Radical: A Possible Astrophysical N-Heterocycle Precursor
}

\author{
Sommer L. Johansen, ${ }^{\dagger}$ Marie-Aline Martin-Drumel, ${ }^{\ddagger}$ and Kyle N. Crabtree ${ }^{*, \dagger}$ \\ $\dagger$ Department of Chemistry, University of California, Davis, One Shields Ave., Davis, CA \\ 95616 USA \\ $\ddagger$ Institut des Sciences Moléculaires d'Orsay, CNRS, Univ. Paris-Sud, Université \\ Paris-Saclay, F-91405 Orsay France \\ E-mail: kncrabtree@ucdavis.edu
}

\section{Abstract}

A fundamental question in the field of astrochemistry is whether the molecules essential to life originated in the interstellar medium (ISM) and, if so, how they were formed. Nitrogencontaining heterocycles are of particular interest because of their role in biology, but to date no N-heterocycle has been detected in the ISM and it is unclear how and where such species might form. Recently, the $\beta$-cyanovinyl radical ( $\mathrm{HCCHCN})$ was implicated in the low temperature gas-phase formation of pyridine. While neutral vinyl cyanide $\left(\mathrm{H}_{2} \mathrm{CCHCN}\right)$ has been rotationally characterized and detected in the ISM, HCCHCN has not. Here we present the first theoretical study of all three cyanovinyl isomers at the $\operatorname{CCSD}(\mathrm{T}) / \mathrm{ANO} 1$ level of theory and the experimental rotational spectra of cisand trans-HCCHCN, as well as those of their ${ }^{15} \mathrm{~N}$ isotopologues, from 5 to $75 \mathrm{GHz}$. The observed spectra are in good agreement with calculations, and provide a basis for further laboratory and astronomical investigations of these radicals.

\section{Introduction}

The discovery of biological molecules such as amino acids, ${ }^{1}$ nucleobases,,${ }^{2,3}$ and vitamins ${ }^{4}$ on meteorites with non-terrestrial isotopic abundances raises the possibility that such species are formed in space. Aromatic, nitrogencontaining heterocycles are a key building block for many of these molecules; however, while many N-containing species have been identified in space, to date N-heterocycles have not.

Potential astrochemical formation pathways of N-heterocycles include processing of icy grain mantles by UV radiation or cosmic rays ${ }^{5}$ and gas-phase pathways involving polymerization of hydrogen cyanide and acetylene. ${ }^{6}$ These gasphase mechanisms are unlikely to explain the abundance of N-heterocycles on meteorites, because this and similar polymerization mechanisms possess large activation barriers and therefore are unlikely to be operative in cold environments that are shielded from UV radiation. If not shielded, lifetimes are proposed to be only hours, as N-heterocycles are highly susceptible to UV photolysis. ${ }^{7}$

Searches for N-heterocycles in a variety of interstellar environments have been unsuccessful: pyridine, quinoline, and isoquinoline have been searched for in circumstellar shells, ${ }^{8}$ pyrimidine in hot molecular cores,${ }^{8,9}$ malonitrile, quinoline, isoquinoline, and pyrimidine in the molecular cloud TMC- $1,{ }^{10}$ and uracil in molecular cloud Sgr B2(N). ${ }^{11}$ These non-detections suggest that if $\mathrm{N}$-heterocycles are produced in these regions, 
they are depleted rapidly by deposition or further chemical processing. However, benzonitrile, an aromatic molecule containing a nitrogen atom, was recently observed in TMC$1,{ }^{12}$ marking the first interstellar detection of a species comparable to an N-heterocycle. The formation pathway of benzonitrile is unknown, but thought to be driven by low temperature radical-neutral reactions in the gas-phase. If so, similar pathways might exist for N-heterocycle formation.

Recently, pyridine was observed as a product of the pyrolysis of vinyl cyanide $\left(\mathrm{C}_{2} \mathrm{H}_{3} \mathrm{CN}\right.$, $\mathrm{VyCN})$ and nitrosobenzene. ${ }^{13}$ While the experiment was done at $1000 \mathrm{~K}$, calculations suggest that pyridine was formed through the barrierless reaction of vinyl cyanide and the $\beta$ cyanovinyl radical (HCCHCN, $\beta-\mathrm{CV})$, which may be feasible at the low temperatures of dense clouds. These environments are shielded from UV radiation and N-heterocycles could have lifetimes long enough to be incorporated into larger species, such as nitrogen containing polycyclic aromatic hydrocarbons, or deposited onto icy grain surfaces.

Radical-neutral formation pathways of 1,4dihydroquinoline and 1,4-dihydroisoquinoline have also recently been proposed. These Nheterocycles were observed as products in the low temperature reaction of 1,3-butadiene and the pyridyl radical, which was calculated to be barrierless. ${ }^{14}$ The authors suggest that pyridine, in the form of the pyridyl radical, is central to forming more complex nitrogen containing polycyclic aromatic hydrocarbons. These studies motivate further work to determine if such mechanisms are indeed operative in dense clouds. However, $\beta-\mathrm{CV}$, one of the proposed pyridine precursors, has not only never been detected in space, but, to the best of our knowledge, has not been spectroscopically characterized at all.

$\mathrm{VyCN}$ has been studied extensively; the first rotational spectrum was published in 1959, ${ }^{15}$ and it has been detected in molecular clouds such as Sgr B2(N) and TMC-1. ${ }^{16,17}$ Of its three radical derivatives, $\alpha$, cis- $\beta$, and trans- $\beta$, (Figure 1$)$ only the $\alpha$-cyanovinyl radical $\left(\mathrm{H}_{2} \mathrm{CCCN}\right.$, $\alpha$-CV) has been characterized experimentally.
Its rotational spectrum in the centimeter and millimeter bands has been reported, ${ }^{18,19}$ as well as its FTIR emission spectrum. ${ }^{20,21}$ The $\beta-\mathrm{CV}$ radical isomers have been considered in experimental and theoretical studies of $\mathrm{VyCN} \mathrm{UV}$ photodissociation ${ }^{22}$ and in the theoretical study of the gas phase reaction of $\mathrm{VyCN}$ and $\mathrm{OH},{ }^{23}$ but no clear observation of these species exists.

Beyond their potential role in interstellar chemistry, CV radicals are likely involved in other chemical processes. Recently, ALMA was used to confirm the presence of $\mathrm{VyCN}$ in $\mathrm{Ti}^{-}$ tan's atmosphere, ${ }^{24}$ where radical-neutral reactions have been proposed to be significant. ${ }^{25}$ $\mathrm{VyCN}$ is also a thermal decomposition product of a variety of plastics, ${ }^{26}$ and there is ongoing research into the most environmentally safe catalytic combustion mechanisms of $\mathrm{VyCN}{ }^{27}$ Both of these processes likely involve radical intermediates.

Here, we present the first ab initio theoretical structures of $\alpha$, cis- $\beta$-, and trans- $\beta-\mathrm{CV}$ calculated at the same level of theory and the first experimental study of the rotational structure of the $\beta$-CV isomers. We have measured the previously unknown rotational spectra of the cisand trans- $\beta$-CV radicals and their nitrogen15 isotopologues using a combination of cavity Fourier-transform microwave (FTMW) spectroscopy and double resonance techniques. Using these experimental frequencies, interstellar searches for both cis- and trans- $\beta-\mathrm{CV}$ can now be undertaken. These results also enable further spectroscopic investigations, as well as kinetics and dynamics experiments.

\section{Theoretical Methods}

Quantum chemical calculations were performed using the CFOUR package ${ }^{29}$ on the $\alpha$-, cis- $\beta$ and trans- $\beta-\mathrm{CV}$ species. Starting geometries for the $\alpha$ and trans- $\beta$ isomers were set to previously published DFT structures. ${ }^{23}$ To the best of our knowledge, the geometry of the cis- $\beta$ $\mathrm{CV}$ isomer has not been previously calculated, so its initial geometry was set identical to trans$\beta$-CV, except the HCCH dihedral angle was rotated by $180^{\circ}$. Calculations were performed at 


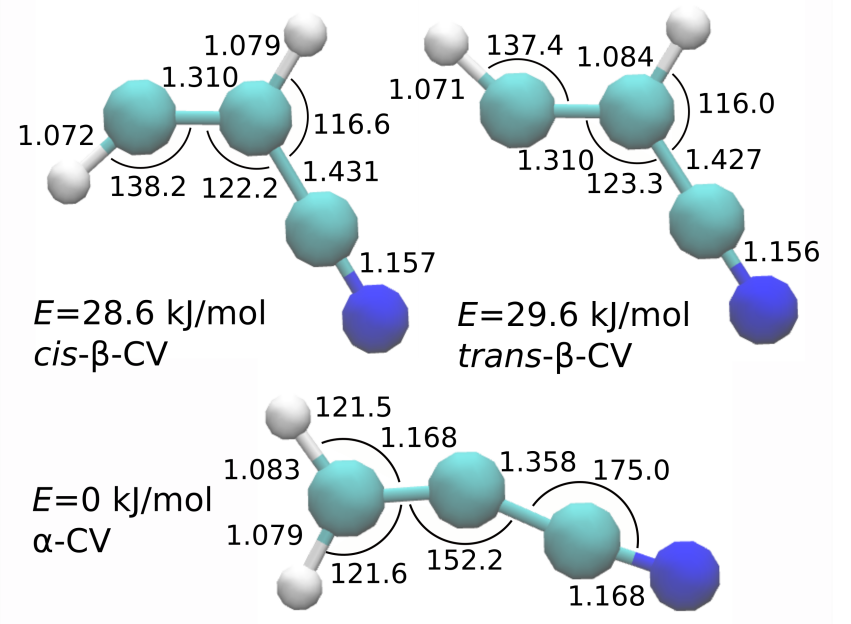

Figure 1: The $\operatorname{CCSD}(\mathrm{T}) / \mathrm{ANO}$ 1 equilibrium structures of cis- $\beta$-CV (top left), trans- $\beta$-CV (top right), and $\alpha-\mathrm{CV}$ (bottom). Bond lengths are in $\AA$ and angles in degrees. Here we define the $\alpha$ carbon as the carbon closest to the highest priority functional group (CN). ${ }^{28}$ The cis and trans isomers are defined by the relative positions of the terminal $\mathrm{H}$ and $\mathrm{CN}$.

the coupled cluster level of theory ${ }^{30}$ with iterative inclusion of single and double excitations and perturbative inclusion of triple excitations $[\operatorname{CCSD}(\mathrm{T})]^{31}$ using atomic natural orbitals (ANO) basis sets. ${ }^{32}$ Equilibrium geometries were first determined at the CCSD/ANO0 level, followed by $\operatorname{CCSD}(\mathrm{T}) / \mathrm{ANO}$, and finally at $\operatorname{CCSD}(\mathrm{T}) / \mathrm{ANO} 1$. While $T_{1}$ diagnostics, ${ }^{33}$ a measure of multireference character in the electronic wavefunction, were higher than is ideal for open-shell coupled cluster calculations, multireference configuration interaction (MRCI) calculations yielded similar results (see Supplemental Information for further detail). Anharmonic vibrational frequencies, zero-point vibrational corrections to the rotational constants, and centrifugal distortion terms were calculated at the $\operatorname{CCSD}(\mathrm{T}) / \mathrm{ANO} 0$ level by second order vibrational perturbation theory ${ }^{34}$ with cubic force fields. Spin-rotation tensor elements (e.g., $\epsilon_{a a}, \epsilon_{b b}, \epsilon_{c c}$ ) were calculated at the $\operatorname{CCSD}(\mathrm{T}) / \mathrm{ANO} 0$ level. First order properties, calculated at the CCSD(T)/ANO1 level, included spin-spin coupling and Fermi contact terms (e.g., $T_{a a}, T_{b b}, a_{F}$ ) for both $\mathrm{H}$ nuclei and the $\mathrm{N}$ nucleus, as well as $\mathrm{N}$-quadrupole coupling terms (e.g., $\left.\chi_{a a}, \chi_{b b}\right)$. All coupled cluster calculations used unrestricted Hartree-Fock as a reference.

\section{Experimental Methods}

VyCN (99\% purity) was purchased from Sigma Aldrich (CAS\#: 107-13-1) and ${ }^{15} \mathrm{~N}$-substituted VyCN (98\% purity) was purchased from Cambridge Isotope Laboratories. The following methods were performed using both normal and isotopically substituted $\mathrm{VyCN}$. Samples were prepared by introducing $1 \mathrm{~g}$ of liquid $\mathrm{VyCN}$ into an evacuated $7 \mathrm{~L}$ gas cylinder which was filled to 200 psi with Ne, yielding $2 \%$ VyCN by pressure before being further diluted with $\mathrm{Ne}$ in a 1:2 ratio in a gas delivery manifold.

This sample was then expanded through a pulsed discharge nozzle ${ }^{35-37}$ operating at $6 \mathrm{~Hz}$, yielding a total flow rate of $15-50$ standard cubic centimeters per minute. The optimal voltage of the discharge source required to produce the $\mathrm{CV}$ species was approximately $1.5 \mathrm{kV}$. From the nozzle, the gas underwent an adiabatic supersonic expansion into a vacuum chamber. This expansion is collision-free and typical rotational temperatures of $\sim 1-2 \mathrm{~K}$ are obtained by the time the sample reaches center of the chamber. ${ }^{36}$

Rotational spectra were measured with a 5 $43 \mathrm{GHz}$ Fabry-Perot cavity Fourier Transform microwave (FTMW) spectrometer, ${ }^{38}$ which consists of two $14 \mathrm{in.} \mathrm{diameter} \mathrm{mirrors} \mathrm{that}$ surround the expanding gas coaxially. Species with rotational transitions near the resonance frequency of the cavity are polarized with a near-resonant microwave pulse, and resultant free induction decays are digitized and processed with a Fourier transform to yield the frequency spectrum. ${ }^{39}$

The program SPCAT $^{40}$ was used to predict the rotational spectra of cis- and trans- $\beta$-CV using our calculated structures and spectroscopic parameters. For each species, a survey was undertaken over a range of $\pm 1 \%$ around the predicted frequency of the fundamental $a$ type transition $\left(N_{K_{a} K_{c}}=1_{01}-0_{00}\right)$. Unknown lines detected in this survey were remeasured 
without the discharge to verify that they disappeared without the plasma, and then with the application of a magnetic field to determine if the carrier was open-shell, as the line profile of transitions of open-shell molecules are significantly altered by the Zeeman effect. The same process was repeated to find the $2_{02}-1_{01}$, $3_{03}-2_{02}$, and $4_{04}-3_{03}$ transitions, all of which were in the range of the cavity spectrometer.

Double resonance (DR) techniques, depicted in Figure 3, were used to verify our assignments of the $a$-type transitions and to find $b$-type transitions out of the range of the spectrometer. In a DR experiment, one line is monitored in the cavity while the sample is irradiated perpendicular to the cavity axis by a second (probe) microwave frequency. When the probe frequency matches that of a transition sharing a quantum state with the monitor line, the intensity of the monitor line is depleted. This technique is described in more detail elsewhere. ${ }^{41}$ The use of various multiplier chains coupled with a DC to $40 \mathrm{GHz}$ microwave synthesizer enabled searches for transitions up to $210 \mathrm{GHz}$.

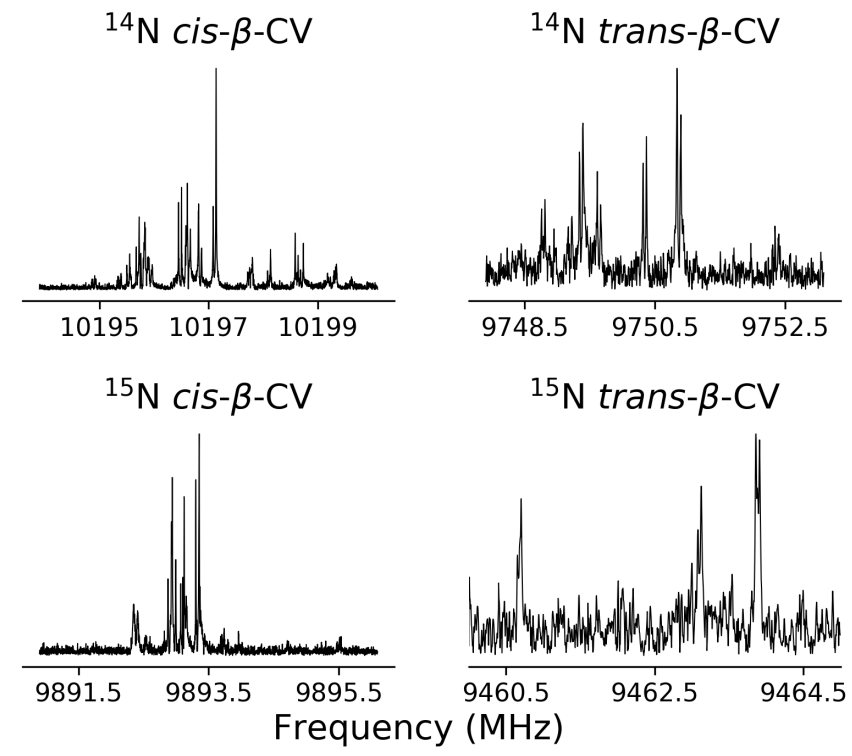

Figure 2: Fundamental a-type transition $\left(N_{K_{a} K_{c}}=1_{01}-0_{00}\right)$ of each isomer and isotopologue of $\beta$-CV. The trans- $\beta$-CV transitions are weaker than those of the cis- $\beta$-CV by about an order of magnitude. Only the strongest trans$\beta$-CV spin-rotation component is shown.

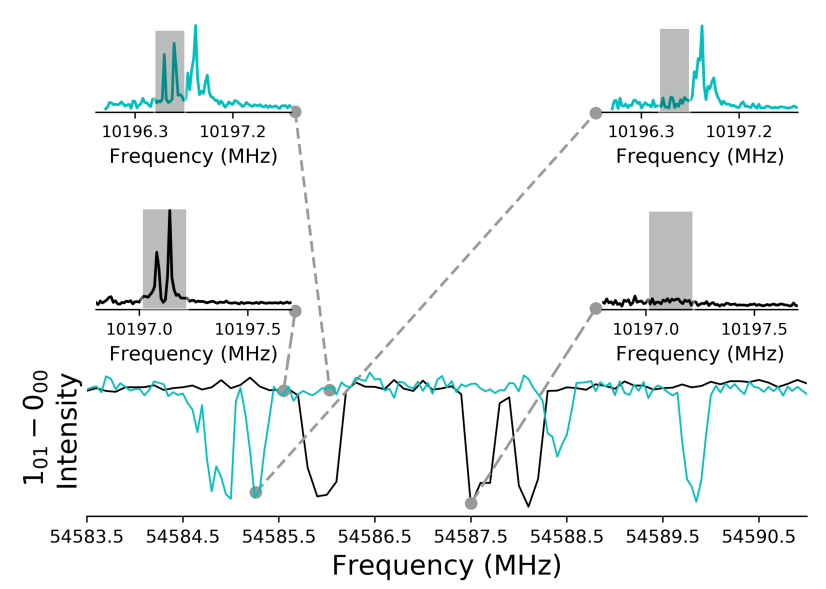

Figure 3: Hyperfine components of the $1_{11}-0_{00}$ $b$-type transition of cis- $\beta$-CV recorded using the DR technique. Insets show the 10196.5 and $10197.1 \mathrm{MHz}$ monitor components of the $1_{01}-0_{00}$ line when the probe frequency was nonresonant (left) and resonant (right). Substantially more lines were detected in the $1_{11}-0_{00}$ transition; here only the lines linked to two of the $1_{01}-0_{00}$ lines are shown.

\section{Results and Discussion}

Of the three $\mathrm{CV}$ isomers, $\alpha$-CV was calculated to be the lowest in energy, with cis- and trans$\beta$-CV $28.6 \mathrm{~kJ} / \mathrm{mol}$ and $29.6 \mathrm{~kJ} / \mathrm{mol}$ higher, respectively (Figure 1). The calculated barrier between cis- and trans- $\beta-\mathrm{CV}$ is $22.2 \mathrm{~kJ} / \mathrm{mol}$ (Figure 4). CCSD(T) and MRCI calculations predict all species to be planar with $C_{s}$ symmetry, although the previous $\alpha$-CV results ${ }^{18}$ suggest that there should be two non-planar geometries with the terminal hydrogens bent out of plane. Computational searches for a nonplanar equilibrium structure of $\alpha-\mathrm{CV}$ with both $\mathrm{CCSD}(\mathrm{T})$ and MRCI methods were unsuccessful. All CV isomers are near-prolate asymmetric tops with $\mu_{a}$ greater than $\mu_{b}\left(\mu_{a}=3.72\right.$, 3.18, 4.91 D and $\mu_{b}=0.18,0.40,1.47 \mathrm{D}$ for $\alpha^{-}$, cis- $\beta$-, and trans- $\beta$-CV, respectively).

The calculated geometries yield equilibrium rotational constants $A_{e}, B_{e}$, and $C_{e}$, and we obtained predictions for the ground state rotational constants $A_{0}, B_{0}$, and $C_{0}$ by including vibration-rotation interaction terms calculated from the anharmonic force fields. To- 


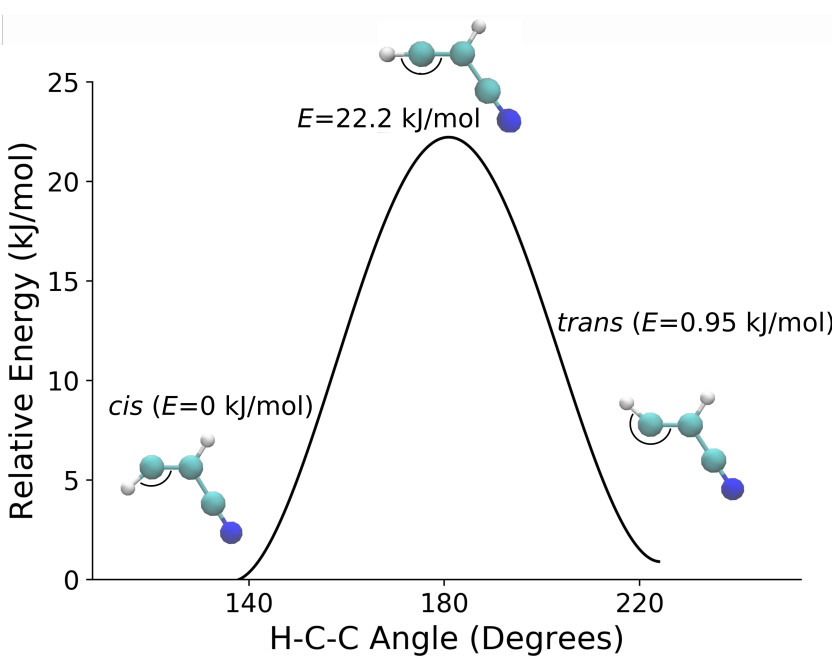

Figure 4: $\operatorname{CCSD}(\mathrm{T}) / \mathrm{ANO} 0$ minimum energy path between cis- and trans- $\beta-\mathrm{CV}$. Equilibrium geometries along the path were calculated with the terminal $\mathrm{H}-\mathrm{C}-\mathrm{C}$ bond angle frozen. All structures are planar, and the transition state $\mathrm{H}-\mathrm{C}-\mathrm{C}$ bond angle is $180^{\circ}$. The barrier height is $22.2 \mathrm{~kJ} / \mathrm{mol}$, and trans- $\beta$-CV is $0.95 \mathrm{~kJ} / \mathrm{mol}$ higher in energy than cis- $\beta$-CV. Here, cis- $\beta$-CV is used as a zero energy reference.

gether with calculated quartic centrifugal distortion constants, we simulated the $N_{K_{a} K_{c}}$ rotational levels for all CV isomers. Each of these levels is expected to be split by spin-rotation and other hyperfine interactions, as will be discussed in more detail later. Full lists of all calculated spectroscopic constants are available in the supplemental information (Table S8).

As a benchmark for our computational work, we compared our calculated $\alpha-\mathrm{CV}$ structure with the previously published experimental rotational spectrum. ${ }^{18}$ The authors of that study detected a series of $a$-type transitions within the $K_{a}=0$ ladder for $\alpha$-CV and its doublydeuterated isotopologue $(\mathrm{d} 2-\alpha-\mathrm{CV})$, and reportedly observed inversion doubling. Because of the lack of any $b$-type transitions or transitions involving $K_{a} \neq 0$ levels, only the rotational constant combination $\left(B_{0}+C_{0}\right) / 2$ was determined for each component of the inversion doublet. As mentioned above, our calculations did not find a nonplanar geometry consistent with the inversion motion, so for comparison we averaged the reported $\left(B_{0}+C_{0}\right) / 2$ values of the two inversion doublet components to com- pare with our ground-state rotational constants for the planar structure. Their value of 4365.6 $\mathrm{MHz}$ for $\alpha$-CV differs from our ab initio calculations by only $0.1 \%\left[\left(B_{0}+C_{0}\right) / 2=4359.9\right.$ $\mathrm{MHz}$. For d2- $\alpha$-CV, the reported $\left(B_{0}+C_{0}\right) / 2$ value is $3953.93 \mathrm{MHz}$, which differs from our calculations by $0.2 \%\left[\left(B_{0}+C_{0}\right) / 2=3944.63\right.$ $\mathrm{MHz}]$

Given the satisfactory agreement of our calculations of $\alpha$-CV with experiment, we began a search for cis- and trans- $\beta$-CV guided by our $a b$ initio structures. We optimized our experimental conditions, such as discharge voltage and flow rates, by maximizing the intensities of the known $\alpha$-CV transitions. In our initial experimental searches, we found putative cisand trans- $\beta-\mathrm{CV} N_{K_{a} K_{c}}=1_{01}-0_{00}$ transitions within $1 \%$ of the frequencies predicted from our quantum chemical calculations. These rotational transitions displayed a complex structure and were affected by the presence of a magnetic field, as expected for open-shell molecules. The cluster of peaks associated with the cis- $\beta$-CV $1_{01}-0_{00}$ transition was of comparable intensity to the corresponding transition of $\alpha$-CV, while the trans- $\beta$-CV transitions were nearly an order of magnitude weaker. Higher $N a$-type transitions for the cis isomer were readily identified at near-harmonic frequencies, while longer integrations were required to find similar transitions for trans- $\beta$-CV. The $2_{02}-1_{01}$ transitions of trans- $\beta-\mathrm{CV}$, found clustered around 19,500.0 $\mathrm{MHz}$, were very close to the $2_{11}-1_{10}$ transitions of vibrationally excited $\mathrm{VyCN}\left(\nu_{11}=1\right)$, which are centered around 19,495.0 $\mathrm{MHz}$.

The fundamental $b$-type transitions $\left(1_{11}-0_{00}\right)$ for both $\beta$ radicals were predicted to lie outside the range of the cavity, requiring searches using DR. These searches covered a large frequency range because the calculated $A$ rotational constant is highly sensitive to inaccuracies in the $\mathrm{H}$ atom positions. Ultimately, the $1_{11}-0_{00}$ transitions were found within $2.3 \%$ of the predictions. Once these lines were assigned, further searches quickly revealed more lines using both FTMW and DR techniques. A total of 84 cis$\beta$-CV lines were measured among 7 rotational transitions. Owing to the lower SNR, we observed 23 trans- $\beta$-CV lines among 6 rotational 
transitions.

The complex structure observed in the spectra arises from the angular momentum coupling within these radicals; rotational transitions are split by spin-rotation coupling between the spin of the unpaired electron and the rotational angular momentum, by quadrupole effects from the ${ }^{14} \mathrm{~N}$ nucleus, and by spin-spin coupling and Fermi-contact interactions due to the three $I>$ 0 nuclei. Simulations of the rotational spectra, including all calculated coupling terms, show that the $1_{01}-0_{00}$ and $1_{11}-0_{00}$ transitions are split into approximately 100 lines each. The number of lines increases significantly with $N$; the $2_{02}-1_{01}$ transitions contain approximately 250 lines. For each rotational transition, there were predicted to be $\sim 30$ lines with intensities within an order of magnitude of the most intense peak. As $N$ increases, the most intense peaks collapse into smaller groups; for instance, in the $9_{09}-8_{08}$ transition of cis- $\beta$-CV near 91 $\mathrm{GHz}$, the most intense peaks are all predicted to fall within about a $1.5 \mathrm{MHz}$ range.

In the experimental cis- $\beta$-CV spectrum, the most intense lines are in a cluster $\sim 5 \mathrm{MHz}$ wide around the rigid-rotor frequencies for all $N$ levels within the range of the cavity. The peaks in the cluster arise from different combinations of angular momenta; however, the splittings induced by each source of angular momentum coupling are comparable in magnitude. In the $a$-type spectrum, this is reflected in the sums of the diagonal elements of the coupling tensors (e.g., $\epsilon_{b b}+\epsilon_{c c}=1.05 \mathrm{MHz}, T_{b b}(\mathrm{H} 1)+T_{c c}(\mathrm{H} 1)$ $=0.54 \mathrm{MHz}$, and $\chi_{b b}+\chi_{c c}=3.88 \mathrm{MHz}$ for $a$-type transitions, etc.). As a result, assigning a full set of angular momentum quantum numbers to each individual transition is challenging, as no dominant source of splitting is obvious. Further discussion about our efforts to assign these quantum numbers and fit the associated spectroscopic constants is provided in the supplemental information. We have therefore only assigned $N, K_{a}$, and $K_{c}$ quantum numbers to the observed transitions of cis- $\beta$-CV.

For trans- $\beta$-CV, the value of $\epsilon_{a a}+\epsilon_{c c}$ (97.38 MHz) is calculated to be much larger than the analogous terms in the other coupling tensors (e.g., $T_{a a}(\mathrm{~N})+T_{c c}(\mathrm{~N})=1.45 \mathrm{MHz}$,
$T_{a a}(\mathrm{H} 1)+T_{c c}(\mathrm{H} 1)=6.24 \mathrm{MHz}$, and $\chi_{a a}+$ $\left.\chi_{c c}=-1.87 \mathrm{MHz}\right)$. Consequently, the complex structure for the fundamental $b$-type transition is predicted to contain two distinct clusters of peaks associated with different spin-rotation components. Like cis- $\beta$-CV, these clusters are also spread over a range of $\sim 5 \mathrm{MHz}$ or less, but they are spaced by about $150 \mathrm{MHz}$ for the $1_{11}-0_{00}$ transition, which is about a factor of 2 larger than predicted from the calculated spinrotation tensor elements. The $a$-type spectrum is more complicated. Our spectrum shows two clusters of lines spaced by about $10 \mathrm{MHz}$, also a bit larger than, but consistent with, the predicted splitting arising from value of $\epsilon_{b b}+\epsilon_{c c}$ $(7.57 \mathrm{MHz})$. However, the spin-spin coupling tensor for the terminal hydrogen (H2) has $T_{b b}$ $+T_{c c}=-40.86 \mathrm{MHz}$, which is expected to produce a $\sim 30 \mathrm{MHz}$ splitting. We have not detected a second component in the $1_{01}-0_{00}$ transition toward either lower or higher frequency, but the SNR of the lines we did observe is low so we cannot rule out that this component is present below the noise level. We have therefore tentatively assigned $N, K_{a}, K_{c}$, and $J$ quantum numbers to all trans- $\beta-\mathrm{CV}$ lines. Complete lists of the frequencies of all observed spectroscopic transitions and their estimated uncertainties for both isomers are available in the supplemental information.

In light of the difficulty in assigning the full complex structure, to determine experimental spectroscopic parameters we simplified the rotational spectrum by approximating the frequency at the center of each assigned cluster of lines, thereby averaging over the hyperfine structure. The uncertainties assigned to these frequencies, of order $\sim 2-3 \mathrm{MHz}$, are estimated based on the observed frequency spread of each cluster, and are therefore substantially larger than the uncertainties on any one peak (of order a few $\mathrm{kHz}$ for lines measured in the cavity, and a few tens of $\mathrm{kHz}$ for DR measurements). These frequencies, shown in Tables 1 and 2, were used along with a standard Watson $A$-reduced effective Hamiltonian ${ }^{42}$ to fit the ground-state rotational constants $A_{0}, B_{0}$, and $C_{0}$ for both isomers, along with $\epsilon_{a a}+\epsilon_{c c}$ and $\epsilon_{b b}+\epsilon_{c c}$ for trans- $\beta$-CV, using quartic CD con- 
stants held at their theoretical values owing to the low $N$ and $K$ quantum numbers in our data set.

Table 3 shows a comparison of the calculated and experimental values for both $\beta-\mathrm{CV}$ species. As expected, the largest deviations arise in the $A_{0}$ values, as these are sensitive to the light atom positions and the anharmonicities associated with their vibrational motion. The $c i s-\beta-\mathrm{CV} B_{0}$ and $C_{0}$ calculated and experimental values agree to within $0.25 \%$, implying that the structure of the heavy atom backbone is calculated quite accurately, while the analogous values calculated for trans- $\beta$-CV larger than the experimental values by almost $1 \%$. This may provide evidence that a second undetected hyperfine component exists at higher frequency than the clusters we observed for each $a$-type rotational transition, which would cause our current fit to underestimate the true values of the $B_{0}$ and $C_{0}$ rotational constants. Alternatively the calculations may be underestimating the $\mathrm{C}=\mathrm{C}-\mathrm{C}$ bond angle or some of the bond lengths along the molecular backbone. Spectroscopy involving ${ }^{13} \mathrm{C}$ isotopic substitution would provide further insight. The experimental spin-rotation tensor elements for trans- $\beta$-CV are larger than the theoretical values by about a factor of 2 ; however, owing to the low SNR, we were not able to observe enough transitions to fit all three diagonal elements simultaneously. Therefore, we constrained the ratio $\epsilon_{b b} / \epsilon_{c c}$ to maintain the $a b i n i$ tio value. Other choices of constraints are possible and would yield different numerical values, so definitive conclusions about the exact magnitudes of the tensor elements cannot be made at present. Nevertheless, the good agreement between the calculated and experimental rotational constants strongly supports the assignments of the detected lines to cis- and trans- $\beta$ CV.

To further verify the identification of these radicals, the spectra of their ${ }^{15} \mathrm{~N}$ isotopologues were obtained. Because the equilibrium geometry of a molecule is unchanged by isotopic substitution (under the Born-Oppenheimer approximation), we used the equilibrium rotational constants (Table 4) to predict the fre- quencies of the fundamental $a$ - and $b$-type transitions of the ${ }^{15} \mathrm{~N}$ species by scaling the observed frequencies of the parent species in the following manner. For the $1_{01}-0_{00}$ transition, we divided the experimental frequency by the calculated $B_{e}+C_{e}$ to obtain a scaling factor, then multiplied the calculated $B_{e}+C_{e}$ value for the ${ }^{15} \mathrm{~N}$ species by that same factor to produce a prediction for its $1_{01}-0_{00}$ frequency. A similar procedure involving $A_{e}+C_{e}$ was used for the $1_{11}-0_{00}$ transition. For trans- $\beta-\mathrm{CV}$, only the lower spin-rotation component of each transition was used in this procedure, as we assume the spin-rotation tensor elements are not significantly changed upon isotopic substitution. In all cases, ${ }^{15} \mathrm{~N}$ transitions were located within $0.02 \%$ of the predicted values, and the $2_{02}-1_{01}$ transitions were also detected (Table 5). The ${ }^{15} \mathrm{~N}$ transitions also showed complex structure that could not be fully assigned, as substituting the $I=1 / 2^{15} \mathrm{~N}$ nucleus for the $I=1^{14} \mathrm{~N} \mathrm{nu-}$ cleus only eliminates the quadrupole coupling tensor, still preserving the spin-spin splitting. Complete line lists for the ${ }^{15} \mathrm{~N}$ isotopologues are given in the supplemental information.

\section{Conclusion}

Here, we report new quantum chemical calculations of the $\alpha-$, cis- $\beta$-, and trans- $\beta$ cyanovinyl radicals at the $\operatorname{CCSD}(\mathrm{T})$ level of theory, and provide the first experimental characterization of the two $\beta-\mathrm{CV}$ radicals by means of their pure rotational spectra. The rotational transitions feature complex structure that is consistent with theoretical predictions, but which cannot be fully assigned at present. Nevertheless, the excellent agreement between the calculated and experimental rotational constants provides strong support for the identifications of these radicals, and the assignments are further confirmed by ${ }^{15} \mathrm{~N}$ isotopic substitution experiments.

The derived rotational constants provide a basis for future spectroscopic studies in the millimeter wave region, where the hyperfine structure is expected to collapse while the spinrotation splitting increases. Such measure- 
Table 1: Rotational transitions of $c i s-\beta-\mathrm{CV}$, in $\mathrm{MHz}$. Frequencies were derived by approximating the centers of the observed clusters of lines (see text). Estimated uncertainties in parentheses are given in units of the last digit.

\begin{tabular}{lcc}
\hline$N_{K_{a}^{\prime}, K_{c}^{\prime}}^{\prime}-N_{K_{a}^{\prime \prime}, K_{c}^{\prime \prime}}^{\prime \prime}$ & Frequency & Obs. - Calc. \\
\hline $1_{01}-0_{00}$ & $10196.67(230)$ & -0.163 \\
$2_{12}-1_{11}$ & $19862.50(230)$ & 0.740 \\
$2_{02}-1_{01}$ & $20389.18(230)$ & 0.336 \\
$3_{03}-2_{02}$ & $30571.41(230)$ & 0.192 \\
$4_{04}-3_{03}$ & $40738.86(230)$ & -0.298 \\
$1_{10}-1_{01}$ & $44922.22(400)$ & 0.058 \\
$1_{11}-0_{00}$ & $54586.15(400)$ & -1.179 \\
$2_{12}-1_{01}$ & $64253.18(400)$ & 0.257 \\
\hline
\end{tabular}

Table 2: Rotational transitions of trans- $\beta-\mathrm{CV}$, in MHz. Frequencies of each spinrotation component were derived by approximating the centers of the observed clusters of lines (see text). Estimated uncertainties in parentheses are given in units of the last digit.

\begin{tabular}{llrc}
\hline$N_{K_{a}^{\prime}, K_{c}^{\prime}}^{\prime}-N_{K_{a}^{\prime \prime}, K_{c}^{\prime \prime}}^{\prime \prime}$ & $J^{\prime}-J^{\prime \prime}$ & \multicolumn{1}{c}{ Frequency } & Obs. - Calc. \\
\hline $1_{01}-0_{00}$ & $3 / 2-1 / 2$ & $9750.23(200)$ & 0.745 \\
$1_{01}-0_{00}$ & $1 / 2-1 / 2$ & $9760.10(200)$ & -0.067 \\
$2_{02}-1_{01}$ & $5 / 2-3 / 2$ & $19500.00(145)$ & -0.696 \\
$2_{02}-1_{01}$ & $3 / 2-1 / 2$ & $19507.00(145)$ & -0.742 \\
$3_{03}-2_{02}$ & $7 / 2-5 / 2$ & $29248.95(200)$ & 0.737 \\
$1_{10}-1_{01}$ & $3 / 2-1 / 2$ & $61161.95(200)$ & -0.706 \\
$1_{11}-0_{00}$ & $3 / 2-1 / 2$ & $70545.39(200)$ & 1.348 \\
$1_{11}-0_{00}$ & $1 / 2-1 / 2$ & $70699.63(200)$ & 0.081 \\
$2_{12}-1_{01}$ & $5 / 2-3 / 2$ & $79932.63(200)$ & -0.723 \\
\hline
\end{tabular}


Table 3: Experimental and calculated Parameters of cis- and trans- $\beta-\mathrm{CV}$, in $\mathrm{MHz}$. Uncertainties in parentheses are given in units of the last digit.

\begin{tabular}{|c|c|c|c|c|c|c|}
\hline \multirow[b]{2}{*}{ Parameter } & \multicolumn{3}{|c|}{ cis } & \multicolumn{3}{|c|}{ trans } \\
\hline & Calc. & Exp. & $\%$ Diff. $^{a}$ & Calc. & Exp. & $\%$ Diff. $^{a}$ \\
\hline$A_{0}$ & 51001.38 & $49757.42(27)$ & -2.50 & 65143.77 & $65918.10(53)$ & 1.17 \\
\hline$B_{0}$ & 5363.23 & $5364.31(16)$ & 0.02 & 5107.83 & $5067.06(59)$ & -0.80 \\
\hline$C_{0}$ & 4844.05 & $4832.54(13)$ & -0.24 & 4728.75 & $4685.71(57)$ & -0.92 \\
\hline $10^{3} \Delta_{N}$ & 3.13 & $3.13^{b}$ & - & 2.15 & $2.15^{b}$ & - \\
\hline $10^{1} \Delta_{N K}$ & -1.10 & $-1.10^{b}$ & - & -1.56 & $-1.56^{b}$ & - \\
\hline$\Delta_{K}$ & 3.03 & $3.03^{b}$ & - & 7.08 & $7.08^{b}$ & - \\
\hline $10^{4} \delta_{N}$ & 6.86 & $6.86^{b}$ & - & 4.19 & $4.19^{b}$ & - \\
\hline $10^{2} \delta_{K}$ & 3.03 & $3.03^{b}$ & - & 2.17 & $2.17^{b}$ & - \\
\hline$\epsilon_{a a}$ & 4.86 & - & - & -99.04 & $-208.15(124)$ & 52.42 \\
\hline$\epsilon_{b b}$ & 5.82 & - & - & -9.23 & $-16.89(111)$ & 45.35 \\
\hline$\epsilon_{c c}$ & -4.77 & - & - & 1.66 & $3.04^{c}$ & - \\
\hline RMS & \multicolumn{3}{|c|}{0.445} & \multicolumn{3}{|c|}{0.398} \\
\hline \multicolumn{7}{|c|}{$\begin{array}{l}{ }^{a}(\text { Exp. }- \text { Calc. }) / \text { Exp. } \times 100 \\
{ }^{b} \text { Held at theoretical values }\end{array}$} \\
\hline
\end{tabular}

Table 4: Calculated equilibrium rotational constants of the ${ }^{14} \mathrm{~N}$ and ${ }^{15} \mathrm{~N}$ cis- and trans$\beta-\mathrm{CV}$ radicals.

\begin{tabular}{lrrrrr}
\hline & \multicolumn{3}{c}{ cis } & & \multicolumn{2}{c}{ trans } \\
\cline { 2 - 3 } \cline { 5 - 6 } Parameter & \multicolumn{1}{c}{${ }^{14} \mathrm{~N}$} & ${ }^{15} \mathrm{~N}$ & & \multicolumn{1}{c}{${ }^{14} \mathrm{~N}$} & ${ }^{15} \mathrm{~N}$ \\
\hline$A_{e}$ & 50602.68 & 50387.46 & & 65002.95 & 64690.40 \\
$B_{e}$ & 5387.26 & 5220.35 & & 5118.28 & 4963.20 \\
$C_{e}$ & 4868.91 & 4730.27 & & 4744.68 & 4609.55 \\
\hline
\end{tabular}

Table 5: Experimental and predicted transition frequencies for the ${ }^{15} \mathrm{~N}$ cis- and trans$\beta-\mathrm{CV}$, in $\mathrm{MHz}$.

\begin{tabular}{lcrrrl}
\hline Species & $N_{K_{a}^{\prime}, K_{c}^{\prime}}^{\prime}-N_{K_{a}^{\prime \prime}, K_{c}^{\prime \prime}}^{\prime \prime}$ & \multicolumn{1}{c}{ Calc. } & Scaled $^{a}$ & \multicolumn{1}{c}{ Exp. } & $\%$ Diff. \\
\hline \multirow{3}{*}{${ }^{15} \mathrm{~N}$ cis- $\beta-\mathrm{CV}$} & $1_{01}-0_{00}$ & 9901.72 & 9891.82 & 9893.39 & 0.02 \\
& $2_{02}-1_{01}$ & 19799.42 & 19779.62 & 19782.12 & 0.01 \\
& $1_{11}-0_{00}$ & 55488.82 & 54240.32 & 54242.73 & 0.004 \\
\hline \multirow{3}{*}{${ }^{15} \mathrm{~N}$ trans- $\beta-\mathrm{CV}$} & $1_{01}-0_{00}$ & 9462.05 & 9463.25 & 9546.35 & 0.02 \\
& $2_{02}-1_{01}$ & 18926.02 & 18924.99 & 19191.04 & 0.004 \\
& $1_{11}-0_{00}$ & 70122.53 & 70116.62 & 69418.09 & 0.01 \\
\hline
\end{tabular}

${ }^{a}$ See text for explanation of scaling.

${ }^{b}($ Exp. - Scaled $) /$ Exp. $\times 100$ 
ments may ultimately assist with interpreting the low- $N$ hyperfine structure observed in the present study. Moreover, our results pave the way for deep astronomical searches for the CV radicals in cold interstellar clouds, where they may participate in barrierless reactions leading to the formation of pyridine, one of the most fundamental N-heterocycles.

\section{Supporting Information}

Discussion and illustrations of attempts to fit the complex rotational structure of $c i s-\beta-\mathrm{CV}$, discussion of $T_{1}$ diagnostics, comparison of MRCI and CCSD $(\mathrm{T})$ calculations, full experimental linelists of both cis- and trans- $\beta$-CV rotational spectra, calculated harmonic and anharmonic frequencies of vibrational modes for cis- $\beta$, trans- $\beta$, and $\alpha-\mathrm{CV}$, lists of all calculated parameters for cis- and trans- $\beta-\mathrm{CV}$, and input files for SPFIT/SPCAT for both $\beta$-CV isomers.

\section{Acknowledgments}

The authors thank Michael C. McCarthy and his group, K. L. Kelvin Lee, Jessie P. Porterfield, and P. Brandon Carroll, at the Harvard Smithsonian Center for Astrophysics for the use of their cavity FTMW spectrometer and helpful discussions. K. L. Kelvin Lee also provided invaluable help with the theoretical work, as did John F. Stanton. Further thanks goes to Kirill Prozument, who provided a copy of Ref. 18, and Harshal Gupta for helpful discussions. S. L. J. was supported by NASA Headquarters under the NASA Earth and Space Science Fellowship Program- Grant 80NSSC18K1110. M.A. M.-D. is thankful to the Programme National "Physique et Chimie du Milieu Interstellaire" (PCMI) of CNRS/INSU with INC/INP co-funded by CEA and CNES for support.

\section{References}

(1) Pizzarello, S.; Huang, Y. The deuterium enrichment of individual amino acids in carbonaceous meteorites: A case for the presolar distribution of biomolecule precursors. Geochimica et Cosmochimica Acta 2005, 69, 599-605.

(2) Martins, Z.; Botta, O.; Fogel, M. L.; Sephton, M. A.; Glavin, D. P.; Watson, J. S.; Dworkin, J. P.; Schwartz, A. W.; Ehrenfreund, P. Extraterrestrial nucleobases in the Murchison meteorite. Earth and Planetary Science Letters 2008, 270, 130-136.

(3) Callahan, M. P.; Smith, K. E.; Cleaves, H. J.; Ruzicka, J.; Stern, J. C.; Glavin, D. P.; House, C. H.; Dworkin, J. P. Carbonaceous meteorites contain a wide range of extraterrestrial nucleobases. Proceedings of the National Academy of Sciences 2011, 108, 13995-13998.

(4) Smith, K. E.; Callahan, M. P.; Gerakines, P. A.; Dworkin, J. P.; House, C. H. Investigation of pyridine carboxylic acids in CM2 carbonaceous chondrites: Potential precursor molecules for ancient coenzymes. Geochimica et Cosmochimica Acta 2014, 136, 1-12.

(5) Materese, C. K.; Nuevo, M.; Sandford, S. A. N- and O-heterocycles produced from the irradiation of benzene and naphthalene in $\mathrm{H}_{2} \mathrm{ONH}_{3}$-containing ices. Astrophysical Journal 2015, 800, 116.

(6) Ricca, A.; Bauschlicher, C. W.; Rosi, M. Mechanisms for the incorporation of a nitrogen atom into polycyclic aromatic hydrocarbon cations. Chemical Physics Letters 2001, 347, 473-480.

(7) Peeters, Z.; Botta, O.; Charnley, S. B.; Kisiel, Z.; Kuan, Y.-J.; Ehrenfreund, P. Formation and photostability of Nheterocycles in space. Astronomy and Astrophysics 2005, 433, 583-590.

(8) Charnley, S. B.; Kuan, Y.-J.; Huang, H.C.; Botta, O.; Butner, H. M.; Cox, N.; Despois, D.; Ehrenfreund, P.; Kisiel, Z.; Lee, Y.-Y. et al. Astronomical searches for nitrogen heterocycles. Advances in Space Research 2005, 36, 137-145. 
(9) Kuan, Y.-J.; Yan, C.-H.; Charnley, S. B.; Kisiel, Z.; Ehrenfreund, P.; Huang, H.C. A search for interstellar pyrimidine. Monthly Notices of the Royal Astronomical Society 2003, 345, 650-656.

(10) Cordiner, M. A.; Charnley, S. B.; Kisiel, Z.; McGuire, B. A.; Kuan, Y.J. Deep K-band observations of TMC-1 with the Green Bank Telescope: Detection of $\mathrm{HC}_{7} \mathrm{O}$, nondetection of $\mathrm{HC}_{11} \mathrm{~N}$, and a search for new organic molecules. The Astrophysical Journal 2017, 850, 187.

(11) Brünken, S.; McCarthy, M. C.; Thaddeus, P.; Godfrey, P. D.; Brown, R. D. Improved line frequencies for the nucleic acid base uracil for a radioastronomical search. Astronomy \& Astrophysics 2006, 459, 317-320.

(12) McGuire, B. A.; Burkhardt, A. M.; Kalenskii, S.; Shingledecker, C. N.; Remijan, A. J.; Herbst, E.; McCarthy, M. C. Detection of the aromatic molecule benzonitrile $\left(\mathrm{c}-\mathrm{C}_{6} \mathrm{H}_{5} \mathrm{CN}\right)$ in the interstellar medium. Science 2018, 359, 202-205.

(13) Parker, D. S. N.; Kaiser, R. I.; Kostko, O.; Troy, T. P.; Ahmed, M.; Sun, B.-J.; Chen, S.-H.; Chang, A. H. H. On the formation of pyridine in the interstellar medium. Physical Chemistry Chemical Physics 2015, 17, 32000-32008.

(14) Parker, D. S. N.; Yang, T.; Dangi, B. B.; Kaiser, R. I.; Bera, P. P.; Lee, T. J. Low temperature formation of nitrogensubstituted polycyclic aromatic hydrocarbons (PANHs) - barrierless routes to dihydro(iso)quinolines. The Astrophysical Journal 2015, 815, 115.

(15) Costain, C. C.; Stoicheff, B. P. Microwave spectrum, molecular structure of vinyl cyanide and a summary of $\mathrm{CC}, \mathrm{CH}$ bond lengths in simple molecules. The Journal of Chemical Physics 1959, 30, 777.

(16) Gardner, F. F.; Winnewisser, G. The detection of interstellar vinyl cyanide (acrylonitrile). The Astrophysical Journal 1975, 195, L127-L130.

(17) Matthews, H. E.; Sears, T. J. The detection of vinyl cyanide in TMC-1. The Astrophysical Journal 1983, 272, 149.

(18) Seiki, K.; Sumiyoshi, Y.; Oshima, Y.; Endo, Y.; Tang, J. Detection of the $\mathrm{H}_{2} \mathrm{CCCN}$ radical by FTMW spectroscopy and observation of the tunneling inversion motion. Bunshi Kozo Sogo Toronkai Koen Yoshishu 2000, 2000, 11.

(19) Prozument, K.; Shaver, R. G.; Ciuba, M. A.; Muenter, J. S.; Park, G. B.; Stanton, J. F.; Guo, H.; Wong, B. M.; Perry, D. S.; Field, R. W. A new approach toward transition state spectroscopy. Faraday Discussions 2013, 163, 33.

(20) Letendre, L.; Dai, H.-L. Structure and vibrational modes of the cyanovinyl radical: A study by time-resolved Fourier transform IR emission spectroscopy. The Journal of Physical Chemistry A 2002, 106, 12035-12040.

(21) Letendre, L. T.; McNavage, W.; Pibel, C.; Liu, D.-K.; Dai, H.-L. Timeresolved FTIR emission spectroscopy of transient radicals. Journal of the Chinese Chemical Society 2005, 52, 677-686.

(22) Guo, J.; Carrington, T.; Filseth, S. V. Energy disposal in $\mathrm{CN}\left(X^{2} \Sigma^{+}\right)$produced in the $157 \mathrm{~nm}$ photodissociation of acrylonitrile. The Journal of Chemical Physics 2001, 115, 8411-8417.

(23) Sun, J.; Wang, R.; Wang, B. Theoretical study on the gas phase reaction of acrylonitrile with a hydroxyl radical. Physical Chemistry Chemical Physics 2011, 13, 16585-16595.

(24) Palmer, M. Y.; Cordiner, M. A.; Nixon, C. A.; Charnley, S. B.; Teanby, N. A.; Kisiel, Z.; Irwin, P. G. J.; Mumma, M. J. ALMA detection and astrobiological potential of vinyl 
cyanide on Titan. Science Advances 2017, 3, e1700022.

(25) Yung, Y. L.; Allen, M.; Pinto, J. P. Photochemistry of the atmosphere of Titan Comparison between model and observations. The Astrophysical Journal Supplement Series 1984, 55, 465.

(26) Levin, B. C. A summary of the NBS literature reviews on the chemical nature and toxicity of the pyrolysis and combustion products from seven plastics: Acrylonitrile-butadiene-styrenes (ABS), nylons, polyesters, polyethylenes, polystyrenes, poly(vinyl chlorides) and rigid polyurethane foams. Fire and Materials 1987, 11, 143-157.

(27) Liu, N.; Yuan, X.; Zhang, R.; Xu, R.; Li, Y. Mechanistic insight into selective catalytic combustion of acrylonitrile $\left(\mathrm{C}_{2} \mathrm{H}_{3} \mathrm{CN}\right)$ : $\mathrm{NCO}$ formation and its further transformation towards $\mathrm{N}_{2}$. Physical Chemistry Chemical Physics 2017, 19, 7971-7979.

(28) Our convention for naming the $\alpha$ and $\beta$ cyanovinyl isomers is the opposite of that in Parker et al. ${ }^{13}$, since they define the alpha carbon as the terminal carbon.

(29) Stanton, J. F.; Gauss, J.; Cheng, L.; Harding, M. E.; Matthews, D. A.; Szalay, P. G. CFOUR, Coupled-Cluster techniques for Computational Chemistry, a quantumchemical program package. With contributions from A.A. Auer, R.J. Bartlett, U. Benedikt, C. Berger, D.E. Bernholdt, Y.J. Bomble, O. Christiansen, F. Engel, R. Faber, M. Heckert, O. Heun, M. Hilgenberg, C. Huber, T.-C. Jagau, D. Jonsson, J. Jusélius, T. Kirsch, K. Klein, W.J. Lauderdale, F. Lipparini, T. Metzroth, L.A. Mück, D.P. O'Neill, D.R. Price, E. Prochnow, C. Puzzarini, K. Ruud, F. Schiffmann, W. Schwalbach, C. Simmons, S. Stopkowicz, A. Tajti, J. Vázquez, F. Wang, J.D. Watts and the integral packages MOLECULE (J. Almlöf and P.R. Taylor), PROPS (P.R. Taylor), ABACUS (T. Helgaker, H.J. Aa. Jensen, P. Jørgensen, and J. Olsen), and ECP routines by A. V. Mitin and C. van Wüllen. For the current version, see http://www.cfour.de.

(30) Bartlett, R. J. Many-body perturbation theory and coupled cluster theory for electron correlation in molecules. Annual Review of Physical Chemistry 1981, 32, 359401.

(31) Raghavachari, K.; Trucks, G. W.; Pople, J. A.; Head-Gordon, M. A fifthorder perturbation comparison of electron correlation theories. Chemical Physics Letters 1989, 157, 479-483.

(32) Almlöf, J.; Taylor, P. R. General contraction of Gaussian basis sets. I. Atomic natural orbitals for first- and second-row atoms. The Journal of Chemical Physics 1987, 86, 4070-4077.

(33) Jayatilaka, D.; Lee, T. J. Open-shell coupled-cluster theory. The Journal of Chemical Physics 1993, 98, 9734-9747.

(34) Mills, I. M. Vibration-rotation structure in asymmetric- and symmetric-top molecules. Molecular Spectroscopy 1972, 115-140.

(35) McCarthy, M. C.; Travers, M. J.; Kovacs, A.; Gottlieb, C. A.; Thaddeus, P. Eight new carbon chain molecules. The Astrophysical Journal Supplement Series 1997, 113, 105-120.

(36) McCarthy, M. C.; Chen, W.; Travers, M. J.; Thaddeus, P. Microwave spectra of 11 polyyne carbon chains. The Astrophysical Journal Supplement Series 2000, 129, 611-623.

(37) McCarthy, M. C.; Martinez, O.; McGuire, B. A.; Crabtree, K. N.; Martin-Drumel, M.-A.; Stanton, J. F. Isotopic studies of trans- and cis- HOCO using rotational spectroscopy: Formation, 
chemical bonding, and molecular structures. The Journal of Chemical Physics 2016, 144, 124304.

(38) Balle, T. J.; Flygare, W. H. Fabry-Perot cavity pulsed Fourier transform microwave spectrometer with a pulsed nozzle particle source. Review of Scientific Instruments 1981, 52, 33-45.

(39) Crabtree, K. N.; Martin-Drumel, M.-A.; Brown, G. G.; Gaster, S. A.; Hall, T. M.; McCarthy, M. C. Microwave spectral taxonomy: A semi-automated combination of chirped-pulse and cavity Fouriertransform microwave spectroscopy. The Journal of Chemical Physics 2016, 144, 124201.

(40) Pickett, H. M. The fitting and prediction of vibration-rotation spectra with spin interactions. Journal of Molecular Spectroscopy 1991, 148, 371-377.

(41) Markov, V. N.; Xu, Y.; Jäger, W.; Jä, W. Microwave-submillimeter wave double-resonance spectrometer for the investigation of van der Waals complexes. Review of Scientific Instruments 1998, 69, 124201.

(42) Watson, J. K. G. Determination of centrifugal distortion coefficients of asymmetric-top molecules. The Journal of Chemical Physics 1967, 46, 1935-1949. 


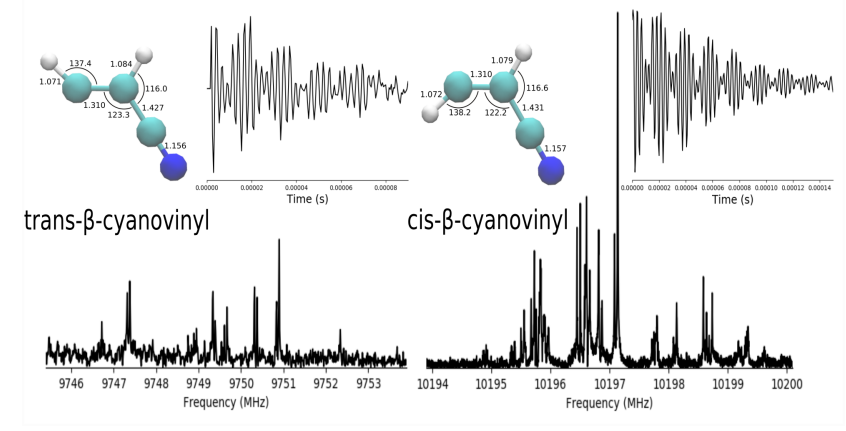

TOC Graphic 